\title{
CONVERGENCE IN PARTIALLY ORDERED GROUPS
}

\author{
by ANDREW WIRTH $\dagger$
}

(Received 31st May 1972)

\begin{abstract}
Relative uniform limits need not be unique in a non-archimedean partially ordered group, and order convergence need not imply metric convergence in a Banach lattice. We define a new type of convergence on partially ordered groups ( $R$-convergence), which implies both the previous ones, and does not have these defects. Further $R$-convergence is equivalent to relative uniform convergence on divisible directed integrally closed partially ordered groups, and to order convergence on fully ordered groups.
\end{abstract}

\section{Introduction}

The open-interval topology is usually not considered a satisfactory one for partially ordered groups (abbreviated to pogroups), because it is discrete for non-fully ordered $l$-groups. We shall indicate, however, that it is of interest if we proceed as follows.

Given a pogroup we consider the family of restrictions of this order, which satisfy an interpolation property and are related in a certain sense to the original order. Each of the open-interval topologies for this family of orders makes the group a Hausdorff topological group. We say a net is $R$-convergent if it is convergent in one of these open-interval topologies. $R$-limits are unique, and in an $l$-group the operations,$+ \vee$ and $\wedge$ are continuous with respect to taking $R$-limits. $R$-convergence is related to relative uniform convergence and order convergence as indicated above, and also it implies convergence in the interval topology. We also determine the relation between $R$-convergence and the inductive limit of the family of open-interval topologies.

\section{Preliminaries}

Let $(G, \leqq)$ be a pogroup. Taking the intervals $(a, b)=\{x: a<x<b\}$, where $a, b \in G, a<b$, as a sub-base, we define the open-interval topology $\mathscr{U}$ on $G$. Denote by $S^{-\mathscr{q} l}$ or $S^{-}$the closure of $S \subseteq G$ in $(G, \mathscr{U})$. We say $(G, \leqq)$ is integrally closed if $n x \leqq y$ for $n=1,2,3, \ldots$ implies that $x \leqq 0$. (G, $\leqq)$ is archimedean if $n x \leqq y$ for all integers $n$ implies that $x=0$. We say $x \in G$ is pseudopositive if $x \quad 0$ and $x+p>0$ for all $p>0$. If $x$ and $-x$ are both pseudopositive, then $x$ is

$\dagger$ This research was supported by a Commonwealth Postgraduate Research Award. The author is grateful to Professors J. B. Miller and G. Birkhoff for their helpful suggestions. 
said to be a pseudozero. If $(G, \leqq)$ has no pseudozeros we write $0<' x$ to mean ' $0<x$ or $x$ is pseudopositive '. Then $(G, \leqq$ ') is a pogroup and we call $\leqq$ ' the associated order. Write $\left(\leqq^{\prime}\right)$ ' (if it exists) as $\leqq$ ".

We say $(G, \leqq)$ satisfies the tight Riesz $(m, n)$ interpolation property (abbreviated $T R(m, n)$ ) (3) if, given $a_{1}, \ldots, a_{m}, b_{1}, \ldots, b_{n} \in G$ with $a_{i}<b_{j}$ for $i=1, \ldots, m, j=1, \ldots, n$, there exists $c \in G$ such that $a_{i}<c<b_{j}$ for $i=1, \ldots, m$, $j=1, \ldots, n$. We say $(G, \leqq)$ is dense if it is $T R(1,1)$. Clearly $T R(m, n)$ is equivalent to $T R(n, m)$ and $T R(2, n)$ implies $T R(m, n)$ for $m \geqq 2$. However $T R(1,2)$ does not imply $T R(2,2)(3)$.

If for every $a>0$, and every positive integer $n$ there exists $b>0$, such that $a>n b$, we say $(G, \leqq)$ contains small elements. We say $G$ is divisible if given $x \in G, n$ a positive integer, there exists $y \in G$ such that $n y=x$. For any other undefined terms see Birkhoff's book (2).

Definition. Let $(G, \preccurlyeq)$ be a non-trivially ordered pogroup. Then $\leqq$ is a compatible tight Riesz order (abbreviated CTRO) on $(G, \preccurlyeq)$ if $(G, \leqq)$ is a nontrivially ordered pogroup, satisfies $T R(1,2)$ and $\varliminf^{\prime}=\preccurlyeq$.

Lemma 1. Let $(G, \leqq)$ be $T R(1,2)$. Then $(G, \leqq, \mathscr{U})$ is a topological group, which is Hausdorff if and only if $(G, \leqq)$ has no pseudozeros. Also $(G, \leqq)$ contains small elements and the family $\{(-a, a): a>0\}$ form a base for $\mathcal{U}$ at 0 . If $(G, \leqq)$ has no pseudozeros and $a<b$, then $(a, b)^{-}=\left\{x: a \leqq^{\prime} x \leqq ' b\right\}$.

The proof is essentially the same as for abelian $T R(2,2)$ groups in (6).

Note. If $(G, \leqq)$ is dense and has no pseudozeros then $\leqq "=\leqq$. So in particular, if $\leqq$ is a CTRO on $(G, \preccurlyeq)$ then $(G, \preccurlyeq)$ has no pseudopositives. If $(G, \leqq)$ is an $l$-group, and $x+p>0$ for all $p>0$, then $0=\bigwedge_{p>0} p \geqq-x$. If $(G, \leqq)$ is an integrally closed divisible isolated pogroup and $x+p / n>0$ for $n=1,2, \ldots$, and $p>0$, then $p>n(-x)$, so $x \geqq 0$. Hence in either case $\leqq \prime=\leqq$.

Lemma 2. There is a one-one correspondence between CTROs on $(G, \preccurlyeq)$ and sets $T$ with the properties:

(i) $T$ is l-directed (i.e. $a, b \in T$ implies that there exists $c \in T$ with $a, b \geqslant c$ ), $T$ is an upper class (i.e. $a \in T$ and $x \geqslant a$ implies that $x \in T$ ) and $\emptyset \neq T \subset G^{+}$,

(ii) $T=T+T$,

(iii) $\bigwedge_{\lessgtr} T=0$,

(iv) $T$ is normal (i.e. $-x+T+x=T$ for all $x \in G$ ).

In fact the set of strictly positive elements of a CTRO satisfies conditions (i)-(iv) and vice versa.

Proof. The proof which can be adapted from that of Theorem 2 in (9) dealing with the abelian $l$-group case, will be omitted. The non-abelian $l$-group case has been considered in (7). 
Note. In (9) we defined a CTRO, $\leqq$ on an abelian non-trivial l-group $(G, \preccurlyeq)$ to be $T R(2,2)$ with $\leqq^{\prime}=\preccurlyeq$ and $(G, \leqq)$ directed. We showed in (9) that conditions (i)-(iii) of Lemma 2 above are necessary and sufficient for a CTRO on an abelian $l$-group. So the definitions are consistent, the one here generalises the previous one.

We will denote a CTRO by $T$ or $\leqq$ as the need arises, and the family of all CTROs by $\mathscr{C}$. In (9) we give sufficient conditions for an abelian $l$-group to have a CTRO; in particular divisibility is such a condition.

Lemma 3. If $(G, \preccurlyeq)$ has a CTRO then $(\mathscr{C}, \subseteq)$ is l-directed. Also $T_{1} \subseteq T_{2}$ if and only if $\mathscr{U}_{1} \subseteq \mathscr{U}_{2}$.

Proof. If $T_{1}, T_{2} \in \mathscr{C}$, let $T_{3}=T_{1}+T_{2}$. If $t_{i} \in T_{i}$ for $i=1,2$, then $t_{1}+t_{2} \geqslant t_{i}$, for $i=1,2$. So by Lemma 2 (i), $T_{3} \subseteq T_{i}$ for $i=1,2$. Since $T_{1}$ and $T_{2}$ are normal, $T_{3}$ is the subsemigroup generated by $T_{1}$ and $T_{2}$. The proof of Theorem 6 in (9) can be adapted to show that $T_{3} \in \mathscr{C}$.

Suppose that $T_{1}, T_{2} \in \mathscr{C}$ and $T_{1} \subseteq T_{2}$. Let $0<{ }_{1} a$, then by Lemma 1 , to show that $\mathscr{U}_{1} \subseteq \mathscr{U}_{2}$ it will suffice to show that $\left\{x:-a<_{1} x<_{1} a\right\}$ is a $\mathscr{U}_{2}$-neighbourhood of 0 . Now there exists $b \in G$, such that $0<_{1} b<_{1} a$, hence $0<{ }_{2} b$. If $y<{ }_{2} b$, then $y<b$, by Lemma 2 (i), and so $y<{ }_{1} a$, also by Lemma 2 (i). So $\left\{y:-b<_{2} y<{ }_{2} b\right\} \subseteq\left\{x:-a<_{1} x<{ }_{1} a\right\}$.

Conversely, let $\mathscr{U}_{1} \subseteq \mathscr{U}_{2}$. If $a>_{1} 0$, then for some $b>_{2} 0,-b{ }_{2} x<{ }_{2} b$ implies that $-a<{ }_{1} x<{ }_{1} a$. Now there exists $c \in G$ such that $b>{ }_{2} c>{ }_{2} 0>_{2}-b$. So $0<{ }_{2} c<{ }_{1} a$. Hence $0<{ }_{2} c \prec a$, and so $0<{ }_{2} a$. Hence $T_{1} \subseteq T_{2}$.

\section{3. $R$-convergence}

Let $(G, \preccurlyeq)$ be a non-trivially ordered pogroup.

Definition. The net $\left\{x_{\alpha}\right\} R$-converges to $x$, i.e. $R$ - $\lim x_{\alpha}=x$, if either $\left\{x_{\alpha}\right\}$ converges to $x$ in the open-interval topology of some CTRO on $\left(G, \preccurlyeq^{\prime}\right)$ or there exists $\alpha_{0}$ such that $x_{\alpha}=x$ for all $\alpha \geqq \alpha_{0}$.

If $\left(G, \preccurlyeq^{\prime}\right)$ has no CTROS, or if $(G, \preccurlyeq)$ has pseudozeros and so $\preccurlyeq^{\prime}$ is not defined, then $R$-convergence is convergence in the discrete topology. If $\left\{x_{\alpha}\right\}$ converges to $x$ in the open-interval topology of a CTRO $T$ we will write this as $T-\lim x_{\alpha}=x$.

Theorem 1. Let $\left\{x_{\alpha}\right\}$ and $\left\{y_{\beta}\right\}$ be nets in $G$ then

(i) $R$ - $\lim x_{\alpha}=x$ and $R$ - $\lim x_{\alpha}=y$ implies $x=y$,

(ii) $x_{\alpha}=x$ for all $\alpha$ implies $R-\lim x_{\alpha}=x$,

(iii) If $\left\{x_{\gamma}\right\}$ is a subnet of $\left\{x_{\alpha}\right\}$ and $R-\lim x_{z}=x$ then $R-\lim x_{\gamma}=x$,

(iv) $R$ - $\lim x_{\alpha}=x$ and $R$-lim $y_{\beta}=y$ implies $R$-lim $\left(x_{\alpha}+y_{\beta}\right)=x+y$,

(v) if $(G, \preccurlyeq)$ is an l-group, $R$-lim $y_{\alpha}=0$ and $\left|x_{\alpha}\right| \preccurlyeq\left|y_{\alpha}\right|$ for all $\alpha$ then $R-\lim x_{\alpha}=0$, 
(vi) if $(G, \preccurlyeq)$ is an l-group then $R-\lim x_{\alpha}=x$ and $R$-lim $y_{\beta}=y$ implies that $R$ - $\lim \left(x_{\alpha} \vee y_{\beta}\right)=x \vee y$, and $R$ - $\lim \left(x_{\alpha} \wedge y_{\beta}\right)=x \wedge y$,

(vii) if $(G, \preccurlyeq)$ is a directed partially ordered real vector space and $\preccurlyeq^{\prime}=\preccurlyeq$, then $\lim \lambda_{n}=\lambda$ and $R$-lim $x_{n}=x$ implies $R$-lim $\lambda_{n} x_{n}=\lambda x$, if and only if,$(G, \preccurlyeq)$ is integrally closed. limit.

Proof. (i)-(v). These follow from Lemmas 1 and 3, and the definition of $R$ -

(vi) The proof of this for $T$-lim in the context of $(G, \preccurlyeq)$, an abelian l-group; is given in (6). The $T$-lim result here is proved similarly, and the rest follows from Lemma 3.

(vii) Let $(G, \preccurlyeq)$ be a directed integrally closed partially ordered vector space and so $\preccurlyeq^{\prime}=\preccurlyeq$, with $\lim \lambda_{n}=\lambda$ and $R$-lim $x_{n}=x$. There exists $y>0, x,-x$, since $(G, \preccurlyeq)$ is directed. Let $T_{1}=\{z: \mu z \geqslant y$ for some $\mu>0\}$; so $y / n \in T_{1}$ for all positive integers $n$, and since $(G, \preccurlyeq)$ is integrally closed, $\wedge T_{1}=0$. So by Lemma 2, $T_{1}$ is a CTRO. Now

$$
\lambda x-\lambda_{n} x_{n}=\left(\lambda-\lambda_{n}\right) x+\lambda_{n}\left(x-x_{n}\right)
$$

and $T$ - $\lim x_{n}=x$ for some CTRO $T(\leqq)$. Also $T_{1}-\lim \left(\lambda-\lambda_{n}\right) x=0$. So by (iv) it only remains to show that $R$ - $\lim \lambda_{n}\left(x-x_{n}\right)=0$. There exists $m \geqq\left|\lambda_{n}\right|$ for all $n$. So given $t \in T$ there exists $u \in T$ such that $m u<t$, by Lemma 1. There exists $k$ such that

$$
-u<x-x_{n}<u \text { for all } n \geqq k .
$$

Hence $-t<-m u<\lambda_{n}\left(x-x_{n}\right)<m u<t$ for all $n \geqq k$. So $T \cdot \lim \lambda_{n}\left(x-x_{n}\right)=0$.

Now suppose that $(G, \preccurlyeq)$ is a directed partially ordered vector space which is not integrally closed and $\preccurlyeq^{\prime}=\preccurlyeq$. Then there exist $a, b \in G$ such that $a \neq 0$, $b \succ 0$ and $n a \preccurlyeq b$ for all positive integers $n$. Let $\lambda_{n}=1 / n, x_{n}=b$, then $R-\lim x_{n}=b$ and $\lim \lambda_{n}=0$. Suppose that $T-\lim b / n=x$ for some CTRO $T$, $x \in G$. Then $T-\lim b /(2 n)=x$ by (iii), and also $T-\lim b /(2 n)=x / 2$. So by (i), $x=0$. Hence $\wedge b / n=\wedge T=0$. But $a \leqslant b / n$ for all $n>0$ and $a \leqslant 0$. Hence $\{b / n\}$ has no $R$-limit.

Note. It follows from Theorem 1 (i), (ii), (iii) that $(G, R$-lim) is a limit space in the sense of Birkhoff (1). Also from Theorem 1 (ii)-(v) it follows that our $\boldsymbol{R}$-convergence is a type of Riesz convergence as defined by Leader (5).

Definition. The net $\left\{x_{\alpha}\right\} 0$-converges to $x$, i.e. 0 -lim $x_{a}=x$, if there exists an increasing net $\left\{y_{\beta}\right\}$ and a decreasing net $\left\{z_{\gamma}\right\}$ such that:

(i) $\vee y_{\beta}=x=\wedge z_{\gamma}$

(ii) given any $\beta$, $\gamma$ there exists $\alpha_{0}$ such that $y_{\beta} \preccurlyeq x_{\alpha} \preccurlyeq z_{\gamma}$ for all $\alpha \geqq \alpha_{0}$.

This is essentially Vulikh's definition (8), which allows the three index sets to be different. One disadvantage of insisting on the same index set for all three nets, as is done by many authors, is that then $0-\lim \left\{x_{a}\right\}_{\alpha \in A}$ may not exist, 
but $0-\lim \left\{x_{\alpha}\right\}_{a \in A}$ and $\alpha \geqq \alpha_{0}$ may exist for some $\alpha_{0} \in A$. We note that if $(G, \preccurlyeq)$ is directed then the two definitions agree for sequences.

Order-convergence appears to be satisfactory if $(G, \preccurlyeq)$ is fully ordered. In particular if $G=R$ then 0 -convergence coincides with the usual metric convergence. However, in the Banach lattice $C[0,1] 0$-convergence does not imply metric convergence. In fact, if $f_{n}(x)=x^{n}, x \in[0,1]$, then $0-\lim f_{n}=0$, but $\left\|f_{n}\right\|=1$.

Definition. The sequence $\left\{x_{n}\right\}$ ru-converges to $x$, i.e. $r u$ - $\lim x_{n}=x$, if there exists a sequence of non-negative integers $\left\{\lambda_{n}\right\}$ and $u>0$ such that $\lim \lambda_{n}=\infty$ and $-u \preccurlyeq \lambda_{n}\left(x-x_{n}\right) \preccurlyeq u$ for all $n$.

This is essentially Leader's definition (5); he in fact defines ru-convergence on l-groups. Our definition agrees with the usual one (2) for directed partially ordered vector spaces.

In a Banach lattic ru-convergence implies metric convergence, and the two are equivalent if the Banach lattice has a strong unit (2). However, ru-limits need not be unique in non-archimedean pogroups. In fact, it can easily be shown that in a divisible isolated pogroup they are unique if and only if the pogroup is archimedean. For example, in RoR (lexicographic order), if $x_{n}=(0,1)$ for all $n$ then $r u-\lim x_{n}=(0,1)$ and $r u-\lim x_{n}=(0,0)$.

Definition. Let $\mathscr{F}$ be the collection of all intersections of finite unions of sets of the form $\{x: a \leqslant x \leqslant b\}$. A set $S \subseteq G$ is closed in the interval topology if $S \cap F \in \mathscr{F}$ for all $F \in \mathscr{F}$.

This is Birkhoff's modification of the Frink interval topology, to sets " possibly without universal bounds" (2).

Theorem 2. Let $\left\{x_{\alpha}\right\}$ be a net in $G$ then

(i) 0-convergence in $(G, \preccurlyeq)$ is non-trivial only if $\preccurlyeq^{\prime}=\preccurlyeq$. If $\preccurlyeq^{\prime}=\preccurlyeq$ then $R$ - $\lim x_{\alpha}=x$ implies 0 - $\lim x_{\alpha}=x$,

(ii) if $(G, \preccurlyeq)$ is fully ordered then $R$-convergence and 0 -convergence are equivalent,

(iii) if $\preccurlyeq^{\prime}=\preccurlyeq$ then $R$ - $\lim x_{n}=x$ implies ru-lim $x_{n}=x$,

(iv) if $(G, \preccurlyeq)$ is divisible isolated and directed and $\preccurlyeq^{\prime}=\preccurlyeq$ then $R$-convergence and ru-convergence are equivalent if and only if $(G, \preccurlyeq)$ is integrally closed,

(v) if $\preccurlyeq^{\prime}=\preccurlyeq$ then $R$ - $\lim x_{\alpha}=x$ implies that $\left\{x_{\alpha}\right\}$ converges to $x$ in the interval topology.

Proof. (i) Suppose that $\preccurlyeq^{\prime}=\preccurlyeq$ and $T$-lim $x_{\alpha}=x$ for some CTRO $T$. Let $y_{-t}=x-t, z_{-t}=x+t$, i.e. $(-T, \preccurlyeq)$ is the index set for these two nets. By Lemma 2, $\vee y_{-t}=\wedge z_{-t}=x$, and in fact 0 - lim $x_{\alpha}=x$. 
Now suppose that $(G, \preccurlyeq)$ contains a pseudopositive, $q$ say. If 0 -convergence is non-trivial, i.e. if there exists a net, not eventually constant, which is 0 convergent, then $\wedge S=0$, where $S$ is some non-empty subset of the strictly positive cone of $(G, \preccurlyeq)$. Now $s \succ-q$ for all $s \in S$, so $0 \geqslant-q$. However $q \neq 0$, so we have the required contradiction.

(ii) Trivial.

(iii) Suppose that $\preccurlyeq^{\prime}=\preccurlyeq$ and $T$ - $\lim x_{n}=x$ for some CTRO $T$. Let $u \in T$ and define $\lambda_{n}$ by

$$
\lambda_{n}=\min \left\{\max \left[m \geqq 0:-u \preccurlyeq m\left(x-x_{n}\right) \preccurlyeq u\right], n\right\} .
$$

By the existence of small elements, $\lim \lambda_{n}=\infty$. So $r u$-lim $x_{n}=x$.

(iv) Let $(G, \preccurlyeq)$ be divisible isolated directed and integrally closed. Then by the note following Lemma $1, \preccurlyeq^{\prime}=\preccurlyeq$. Suppose that $-u \preccurlyeq \lambda_{n} x_{n} \preccurlyeq u$ for all $n$, with $u \succ 0$ and $\lim \lambda_{n}=\infty$. Let $T=\{t: k t \geqslant u$ for some positive integer $k\}$. Then $T$ is a CTRO (cf. proof of Theorem 1 (vii)). If $t \in T$ then for some positive integer $m, m t \geqslant u$, so

$$
-t<-\frac{t}{2} \preccurlyeq-\frac{u}{2 m} \preccurlyeq \frac{\lambda_{n}}{2 m} x_{n} \preccurlyeq \frac{u}{2 m} \preccurlyeq \frac{t}{2}<t \text { for all } n .
$$

Since $\lim \lambda_{n}=\infty$ and $(G, \preccurlyeq)$ is isolated, it follows that $-t<x_{n}<t$ for all $n$ sufficiently large. So $T-\lim x_{n}=0$.

If $(G, \preccurlyeq)$ is divisible directed and not integrally closed and $\preccurlyeq^{\prime}=\preccurlyeq$, then with the notation of the second part of the proof of Theorem 1 (vii) ru-lim $b / n=0$ whereas $R$-lim $b / n$ does not exist.

(v) Suppose that $\preccurlyeq^{\prime}=\preccurlyeq$. We show that if $T(\leqq)$ is a CTRO and $S$ is closed in the interval topology then $S$ is $T$-closed. Let $\left\{x_{\alpha}\right\} \subseteq S$ with $T$ - $\lim x_{\alpha}=x$, we show that $x \in S$. Let $t \in T$ then for some $\alpha_{0}, x_{\alpha} \in S \cap\{y: x-t \preccurlyeq y \preccurlyeq x+t\}$ for all $\alpha \geqq \alpha_{0}$. So in fact it will suffice to show that $\{x: a \preccurlyeq x \preccurlyeq b\}$ is a $T$ closed set. If $\left\{y_{\beta}\right\} \subseteq\{x: a \leqslant x \leqslant b\}$ and $T$-lim $y_{\beta}=y$, then $z>y$ implies that $z>y_{\beta}$ for some $\beta$, since $\{x: x<z\}$ is a $T$-neighbourhood of $y$. So $z>y$ implies that $z>a$, hence $y \geqslant a$. Similarly it can be shown that $y \preccurlyeq b$. Hence $\{x: a \leqslant x \leqslant b\}$ is $T$-closed.

It is well known that 0 -convergence and ru-convergence need not be convergence with respect to a topology. The following theorem determines when $R$-convergence is convergence with respect to a topology.

Theorem 3. If $\mathscr{C}$, the set of all CTROs on $\left(G, \preccurlyeq^{\prime}\right)$, is non-empty, then the following are equivalent:

(i) $R$-convergence is convergence with respect to a topology,

(ii) $R$-convergence is convergence in the inductive limit of the CTROs,

(iii) $(\mathscr{C}, \subseteq)$ contains a smallest element. 
Proof. (i) $\Rightarrow$ (ii). Write $T_{1} \preccurlyeq T_{2}$ if $T_{1} \supseteq T_{2}$. Then $(\mathscr{C}, \preccurlyeq)$ is $u$-directed (the dual of $l$-directed), by Lemma 3 . If $T_{a}, T_{\beta} \in \mathscr{C}$, define

$$
\theta_{\alpha \beta}:\left(G, \mathscr{U}_{\alpha}\right) \rightarrow\left(G, \mathscr{U}_{\beta}\right),
$$

by $\theta_{\alpha \beta}(x)=x$ for all $x \in G$. Then if $T_{\alpha} \preccurlyeq T_{\beta}, \theta_{\alpha \beta}$ is continuous by Lemma 3 . Also $\lim \left(G, \mathscr{U}_{\alpha}\right)$ is $G$ with the strongest topology which is at least as weak as all

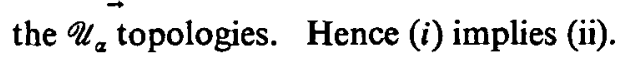

(i) $\Rightarrow$ (iii). If $T_{i} \preccurlyeq T_{j}$ write $i \preccurlyeq j$, and let $\mathscr{C}=\left\{T_{i}: i \in I\right\}$. Then $\{i, \preccurlyeq\}_{i \in I}$ and $\{-t, \preccurlyeq\}_{t \in T_{i}}$ are $u$-directed by Lemmas 3 and 2 , respectively. If $i \in I$ and $t \in T_{i}$, let $y(i,-t)=t$, then

$$
R-\lim _{i \in I} R-\lim _{-t \in-T_{i}} y(i,-t)=0 .
$$

If $R$-convergence is convergence with respect to a topology then by a theorem on iterated limits (4),

$$
R-\lim _{\Gamma} z(i, \ldots,-t, \ldots)=0
$$

where

$$
z(i, \ldots,-t, \ldots)=y(i,-t) \text { and } \Gamma=I \times \prod_{i \in I}\left(-T_{i}\right) .
$$

So for some

$$
T_{0} \in \mathscr{C}, T_{0}-\lim _{\Gamma} z(i, \ldots,-t, \ldots)=0 .
$$

Let $t \in T_{0}$, then there exists $i_{0} \in I$ such that for each $i \geqslant i_{0}, t>u_{i}$, where $u_{i}$ is some element of $T_{i}$, and $\leqq$ refers to $T_{0}$. So by Lemma 2 (i), $t \in T_{i}$ for all $i \geqslant i_{0}$. If $j \in I$, then there exists $i \in I$ such that $i \geqslant j, i_{0}$, since $(I, \preccurlyeq)$ is $u$-directed. So $t \in T_{i}$ and $T_{i} \subseteq T_{j}$, hence $t \in T_{j}$, and so $T_{0} \subseteq T_{j}$ for all $j \in I$. Thus $T_{0}$ is the smallest element of $(C, \subseteq)$.

(ii) $\Rightarrow$ (i), and (iii) $\Rightarrow$ (i) are obvious.

In Lemma 4 of $(9)$ it is shown that if $(G, \preccurlyeq)$ is a divisible integrally closed $l$-group then $(G, \preccurlyeq)$ has a smallest CTRO if and only if $(G, \preccurlyeq)$ has a strong unit.

\section{REFERENCES}

(1) G. BIRKhoff, What can lattices do for you?, Trends in Lattice Theory (ed. by J. C. Abbott, Van Nostrand Reinhold, New York, 1970), 1-38.

(2) G. BrRKhoff, Lattice Theory, 3rd edition (Amer. Math. Soc., Providence, 1967), 250-251 and 366-370.

(3) N. Cameron and J. B. Miller, Topology and axioms of interpolation in partially ordered spaces, Preprint, Monash University (1971).

(4) J. L. Kelley, General Topology (Van Nostrand, Princeton, 1955), 69.

(5) S. Leader, Sequential convergence in lattice groups, Problems in Analysis (ed. by R. C. Gunning, Princeton U.P., Princeton, 1970), 273-290. 
(6) R. J. Loy and J. B. Miller, Tight Riesz groups, J. Austral. Math. Soc., 13 (1972), 224-240.

(7) N. R. Rellly, Compatible tight Riesz orders and prime subgroups, Preprint, Simon Fraser University (1971).

(8) B. Z. VuLIKH, Introduction to the theory of partially ordered spaces (WoltersNoordhoff, Groningen, 1967), 29.

(9) A. WIRTH, Compatible tight Riesz orders, J. Austral. Math. Soc. (to appear).

MONASH UNIVERSITY

Clayton

VICTORIA 